\title{
Observation of charm mixing at CDF
}

\section{Paolo Maestro*}

On behalf of the CDF collaboration

Department of Physics, University of Siena and INFN, via Roma 56, 53100 Siena, Italy

E-mail: paolo.maestro@pi.infn.it

\begin{abstract}
We report on the observation of $D^{0}-\bar{D}^{0}$ oscillations by measuring the time-dependent ratio of yields for the rare decay $D^{0} \rightarrow K^{+} \pi^{-}$to the favored decay $D^{0} \rightarrow K^{-} \pi^{+}$at the Collider Detector at Fermilab (CDF). Using $9.6 \mathrm{fb}^{-1}$ of integrated luminosity of $\sqrt{s}=1.96 \mathrm{TeV} p \bar{p}$ collisions recorded in the full CDF Run II, the signals of $7.6 \times 10^{6} D^{0} \rightarrow K^{-} \pi^{+}$and $33 \times 10^{3} D^{0} \rightarrow K^{+} \pi^{-}$decays are reconstructed in $D^{*}$-tagged events, with proper decay times between 0.75 and 10 mean $D^{0}$ lifetimes. We measure the mixing parameters $x^{\prime 2}=(0.08 \pm 0.18) \times 10^{-3}, y^{\prime}=(4.3 \pm 4.3) \times 10^{-3}$, and $R_{D}=(3.51 \pm 0.35) \times 10^{-3}$. Our results are consistent with standard model expectations and similar results from proton-proton collisions and exclude the no-mixing hypothesis with a significance equivalent to 6.1 standard deviations.
\end{abstract}

14th International Conference on B-Physics at Hadron Machines, April 8-12, 2013

Bologna, Italy

*Speaker. 


\section{Introduction}

Neutral mesons can oscillate into their antiparticles because they are produced in flavor eigenstates which are different from eigenstates with defined mass and lifetime. This quantum-mechanical oscillation is referred as mixing and can be characterized by the parameters $x=\Delta m / \Gamma$ and $y=$ $\Delta \Gamma / 2 \Gamma$, where $\Delta m$ is the mass difference, $\Delta \Gamma$ is the decay width difference, and $\Gamma$ is the mean decay width of the mass eigenstates. The process is well established for $K^{0}, B^{0}$, and $B_{s}^{0}$ mesons [1] and its study provides important information about electroweak interactions and the Cabibbo-KobayashiMaskawa (CKM) matrix, as well as the virtual particles that are exchanged in the mixing process itself. Evidence of $D^{0}-\bar{D}^{0}$ mixing was reported in recent years by the experiments Belle [2], Babar $[3,4]$ and CDF [5] and it was observed only in 2012 by LHCb [6]. Even if standard model (SM) calculations of the $D^{0}-\bar{D}^{0}$ mixing rate are affected by significant theoretical uncertainties, this process is expected to be much slower (i.e. $|x|,|y| \leq 10^{-3}$ ) than the $B$ and $K$ oscillations. However NP particles could enhance the mixing rate, thus providing indirect evidence for physics beyond the SM $[7,8]$. Then, it is of great interest to establish conclusively $D^{0}-\bar{D}^{0}$ mixing in a specific decay channel and improve the precision of the measurement of the mixing parameters.

\subsection{Charm mixing in the $D^{0} \rightarrow K^{+} \pi^{-}$channel}

Charm mixing can be searched by measuring the time dependence of the rate of the rare $D^{0} \rightarrow K^{+} \pi^{-}$decay (including its charge-conjugate). This decay can arise from the oscillation of a $D^{0}$ state to a $\bar{D}^{0}$ state, followed by a Cabibbo-favored (CF) $D^{0} \rightarrow K^{-} \pi^{+}$decay, or from a doublyCabibbo suppressed (DCS) $D^{0}$ decay. Under the assumption that CP is conserved and the mixing parameters are small $(|x|,|y| \ll 1)$, the ratio $R$ of $D^{0} \rightarrow K^{+} \pi^{-}$to $D^{0} \rightarrow K^{-} \pi^{+}$decay rates can be approximated by [1]

$$
R(t)=R_{D}+\sqrt{R_{D}} y^{\prime} t+\frac{x^{\prime 2}+y^{\prime 2}}{4} t^{2}
$$

where $t$ is the proper decay time expressed in units of mean $D^{0}$ lifetime. $R_{D}$ is the DCS decay rate relative to the CF rate, while the parameters $x^{\prime}$ and $y^{\prime}$ are linear combinations of $x$ and $y$ according to the relations $x^{\prime}=y \sin \delta_{K \pi}+x \cos \delta_{K \pi}$ and $y^{\prime}=y \cos \delta_{K \pi}-x \sin \delta_{K \pi}$, where $\delta_{K \pi}$ is the strong interaction phase difference between the DCS and CF amplitudes. In the absence of mixing, $x^{\prime}=y^{\prime}=0$ and $R(t)=R_{D}$.

The experimental method to identify the flavor of the charmed meson at production exploits the strong-interaction decays $D^{*+} \rightarrow \pi^{+} D^{0}, D^{*-} \rightarrow \pi^{-} \bar{D}^{0}$. The relative charges of the soft (lowmomentum) tagging pion from $D^{*}$ decay and the pion from $D^{0}$ decay determine whether the decay chain is right-sign (RS, like charge) or wrong-sign (WS, opposite charge). RS processes include mainly CF decays, while DCS and mixing decays contribute to WS processes.

\section{Analysis}

Our measurement uses the full data set corresponding to an integrated luminosity of $9.6 \mathrm{fb}^{-1}$ recorded by the CDF II detector at the Tevatron in $p \bar{p}$ collisions at $\sqrt{s}=1.96 \mathrm{TeV}$. We reconstruct the WS $D^{*+} \rightarrow \pi^{+} D^{0}\left(\rightarrow K^{+} \pi^{-}\right)$, and the RS $D^{*+} \rightarrow \pi^{+} D^{0}\left(\rightarrow K^{-} \pi^{+}\right)$decay chains and measure the time dependence of their rates ratio. The components of the CDF II detector most relevant 
for this analysis are the multi-wire drift chamber (COT) and the silicon microstrip vertex detector located inside a solenoid, which provides a 1.4 T magnetic field [9].

\subsection{Data selection}

The events for this analysis are selected online by a trigger system [10] which identifies pairs of oppositely charged particle tracks from a decay vertex detached by at least $200 \mu \mathrm{m}$ from the beamline. In the off-line analysis, the tracks satisfying the trigger requirements are considered with both $K^{-} \pi^{+}$and $\pi^{-} K^{+}$interpretations in order to reconstruct $D^{0}$ candidates. Minimal requirements on the momenta and impact parameters of the tracks and the displacement of the reconstructed $D^{0}$ decay vertex are imposed. A low-momentum tagging pion track is combined with the $D^{0}$ candidate to form a $D^{*}$ candidate. To reduce the contribution of $D^{*}$ mesons produced from $b$-hadron decays, $D^{0}$ candidate are required to have an impact parameter $d_{0}<60 \mu \mathrm{m}$. RS $D^{0}$ decays incorrectly reconstructed as WS decays, because the kaon and pion assignments are mistakenly interchanged, represent a large background to the WS signal. Two selection cuts have been applied to reduce this background. WS candidates with RS $K \pi$ invariant mass reconstructed within $20 \mathrm{MeV} / c^{2}$ of the known $D^{0}$ mass are removed. This cut retains $78 \%$ of the WS signal, and rejects $96.5 \%$ of the RS $D^{0}$ decays with incorrect mass assignment. A second cut exploits the $\mathrm{K} / \pi$ separation based on the measurements of the ionization energy loss in the COT [10]. The combination of these two cuts greatly reduces the mis-assigned RS background improving the WS signal over background ratio by a factor $\sim 100$.

\subsection{Signal extraction}

The reconstructed RS and WS condidates are classified into 20 intervals of proper decay time $t$ which is determined (normalized to the mean $D^{0}$ lifetime $\tau=410.1 \mathrm{fs}$ ) as $t=m_{D^{0}} L_{x y} /\left(p_{T} \tau\right)$, where $m_{D^{0}}=1.8648 \mathrm{GeV} / c^{2}$ is the known $D^{0}$ mass [1], $L_{x y}$ is the transverse $D^{0}$ decay length, and $p_{T}$ its transverse momentum. $D^{0}$ candidates in each time bin are further divided into 60 bins of mass difference $\Delta M \equiv M(K \pi \pi)-M(K \pi)-M(\pi)$, with equal size $0.5 \mathrm{MeV} / \mathrm{c}^{2}$. For each of the resulting $1200 \mathrm{WS}$ and $1200 \mathrm{RS}$ bins, the $D^{0}$ signal yield is determined by fitting the corresponding binned distribution of the $\mathrm{K} \pi$ invariant mass $M_{K \pi}$. The signal shape is modeled by the sum of two Gaussian functions with a low-mass tail, and the combinatoric background by an exponential function. A Gaussian term is included in the WS fit to model the residual background from misidentified RS decays, with shape determined from the data. The $D^{*}$ signal for each time bin is determined from a $\chi^{2}$ fit of the $D^{0}$ signal yield versus $\Delta M$. The signal shape is modeled by a double-Gaussian and an asymmetric tail function, the background shape by the product of a power-law and an exponential function. The amplitudes of the signal and background and the background shape parameters are determined independently for all $M_{K \pi}$ and $\Delta M$ fits, while the signal shape is fixed to the RS timeintegrated shape. The $D^{*}$ fit procedure for the time-integrated $\Delta M$ distributions is shown in Fig. 1. The fitted RS and WS signal yields are about $7.6 \times 10^{6}$ and $33 \times 10^{3}$, respectively.

\subsection{WS/RS yield ratio}

The measured ratio $R_{m}$ of WS to RS signal yields in the 20 time intervarls is shown in Fig. 2. Since the measured yields include the contribution of $D^{*}$ mesons produced from $b$-hadron decays, 


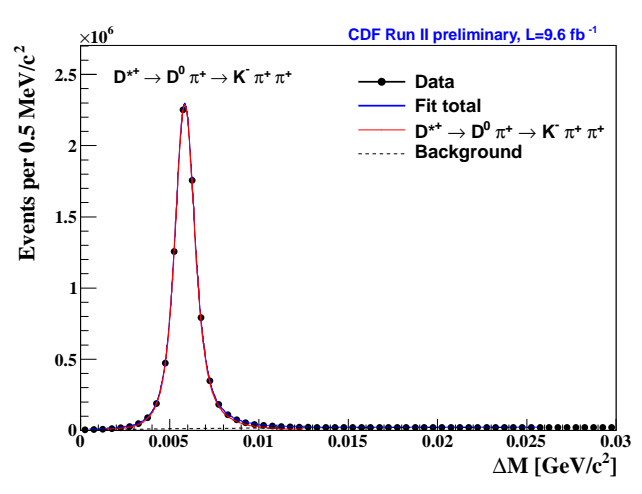

(a)

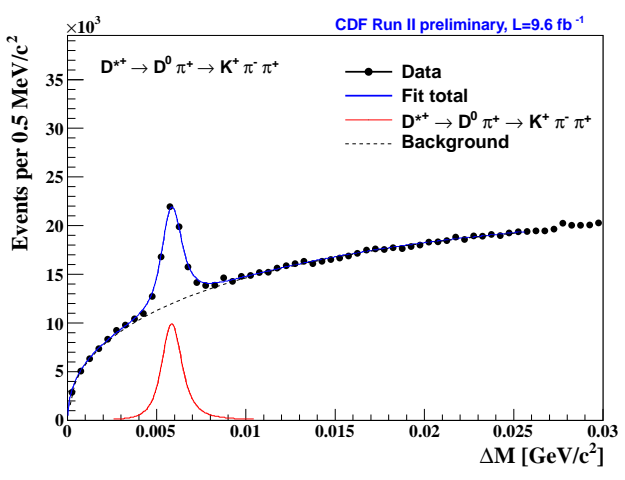

(b)

Figure 1: Time-integrated $\Delta M$ distribution for (a) RS and (b) WS decays, with fit curves superimposed.

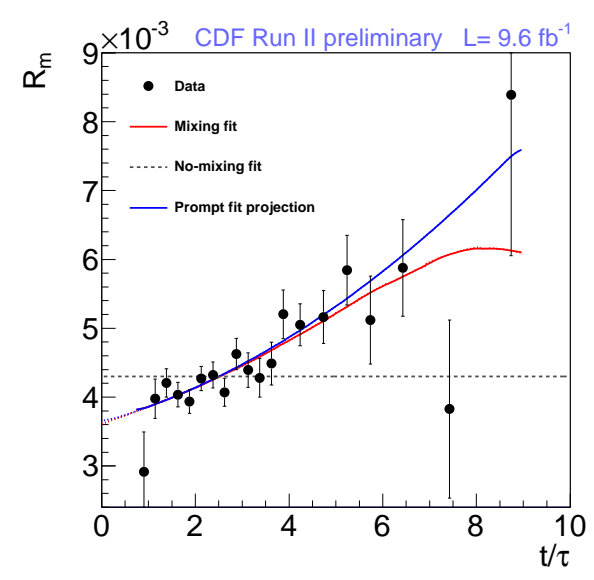

Figure 2: Measured ratio of WS to RS signal yields as a function of normalized proper decay time.

the time dependence of $R_{m}$ is different from that of the WS/RS ratio of prompt decays $R$ (Eq. 1.1). The expected value of $R_{m}$ in a given time bin can be factorized as the product of $R$ by a correction factor due to the non-prompt production

$$
R_{m}^{\text {pred }}(t)=R(t)\left[1+f_{B}(t)\left(\frac{R_{B}(t)}{R(t)}-1\right)\right]
$$

where $f_{B}(t)$ is the fraction of non-prompt RS $D^{*}$ decays and $R_{B}(t)$ is the WS/RS ratio of nonprompt $D^{*}$ decays with measured decay time $t$. For non-prompt decays, the measured decay time is the sum of the decay times of the beauty particle parent and the $D^{0}$ daughter. The function $R_{B}(t)$ is calculated by weighting $R(t)$ with the decay-time distributions of non-prompt $D^{0}$ decays obtained from a full detector simulation. The function $f_{B}(t)$ is determined from data by fitting the $d_{0}$ distributions of RS $D^{*}$ decays in each time bin. These are characterized by a peak at small $d_{0}$ due to the prompt component, and a broad distribution extending to large $d_{0}$ due to the non-prompt component, as shown in Fig. 3(a). Both the prompt and non-prompt components are modeled with the sum of two Gaussians. The time dependence of $f_{B}$ in the region $d_{0}<60 \mu \mathrm{m}$ is parametrized by a 4-degree polynomial (Fig. 3(b)). 


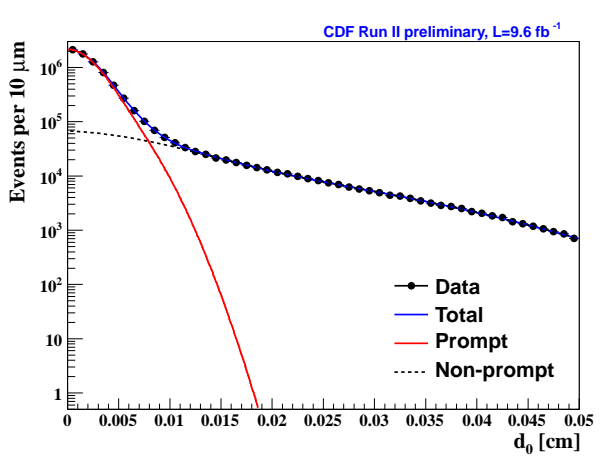

(a)

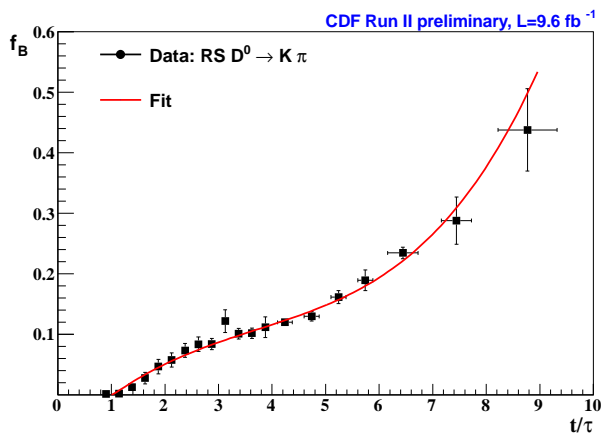

(b)

Figure 3: (a) Distribution of transverse impact parameter $d_{0}$ for $\operatorname{RS} D^{0}$ candidates for all the time bins. (b) Fraction of non-prompt RS $D^{*}$ decays as a function of proper decay time.

\begin{tabular}{|c|c|c|c|ccc|}
\hline Fit type & $\chi^{2} / \mathrm{ndf}$ & Parameter & $\begin{array}{c}\text { Fitted values } \\
\times 10^{-3}\end{array}$ & \multicolumn{3}{|c|}{ Correlation coefficient } \\
& & & $R_{D}$ & $y^{\prime}$ & $x^{\prime 2}$ \\
\hline Mixing & $16.91 / 17$ & $R_{D}$ & $3.51 \pm 0.35$ & 1 & -0.967 & 0.900 \\
& & $y^{\prime}$ & $4.3 \pm 4.3$ & & 1 & -0.975 \\
& & $x^{\prime 2}$ & $0.08 \pm 0.18$ & & & 1 \\
\hline No-mixing & $58.75 / 19$ & $R_{D}$ & $4.30 \pm 0.06$ & & & \\
\hline
\end{tabular}

Table 1: Mixing parameter results. The uncertainties include statistical and systematic components.

The mixing parameters $R_{D}, y^{\prime}$, and $x^{\prime 2}$ are found by minimizing the $\chi^{2}$ function

$$
\chi^{2}=\sum_{i=1}^{20}\left[\frac{R_{m}\left(t_{i}\right)-R_{m}^{\text {pred }}\left(t_{i}\right)}{\sigma_{i}}\right]^{2}+C_{B}+C_{H}
$$

where $\sigma_{i}$ is the uncertainty on $R_{m}\left(t_{i}\right)$ and $C_{B}$ and $C_{H}$ are Gaussian constraints to the parameters describing $f_{B}(t)$ and $R_{B}(t)$, respectively.

We investigated extensively systematic uncertainties due to a number of possible sources including: detector charged track asymmetries, uncertainties in the signal shapes used to fit $M_{K \pi}$ and $\Delta M$ distributions and in the shape of non-prompt component used to fit the $d_{0}$ distributions, background due to $D^{+} \rightarrow K^{-} \pi^{+} \pi^{+}$and partially reconstructed charm decays, sensitivity of $R_{B}(t)$ on the simulated decay time distributions of non-prompt $D^{0}$. All these effects were found to be small compared to the mixing parameter errors derived from the fit.

\subsection{Result}

The fitted values of the mixing parameters are reported in Table 1. The function $R_{m}^{\text {pred }}(t)$ and the prompt component $R(t)$ as determined by the fit are shown in Fig. 2. They differ at large $t$ due to the effect of non-prompt $D^{*}$ production. A fit assuming no-mixing, i.e. $y^{\prime}=x^{\prime 2}=0$, is also shown and is clearly incompatible with the data. By calculating the Bayesian probability contours in the $x^{\prime 2}-y$ parameter space (Fig. 4(a)), we exclude the no-mixing hypothesis at the level of 6.1 Gaussian 


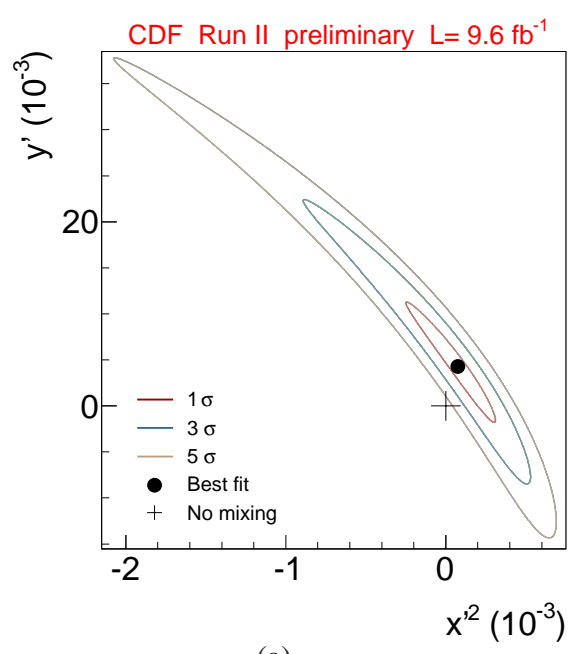

(a)

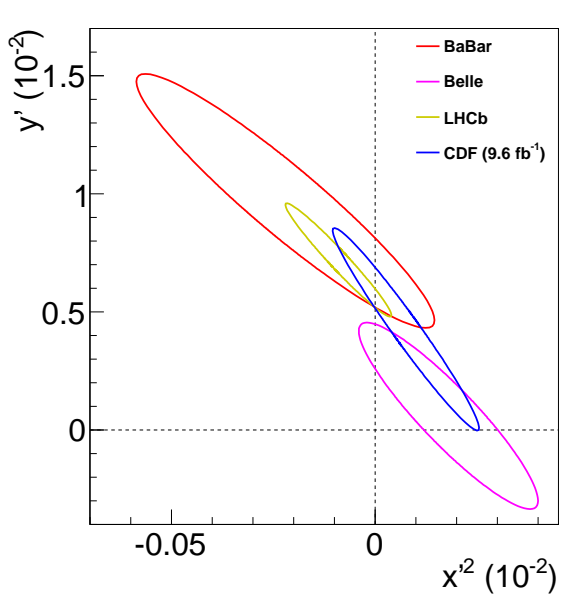

(b)

Figure 4: (a) Bayesian probability contours in $x^{\prime 2}-y^{\prime}$ parameter space. (b) Comparison of $1 \sigma$ contours between CDF (this work), Belle [2], Babar [3, 4] and LHCb [6].

standard deviations. Our results are consistent with SM calculations [7] and measurements from other experiments, as shown by comparing the $1 \sigma x^{\prime 2}-y$ contours in Fig. 4(b), and have similar precision to the recent $\mathrm{LHCb}$ observation [6].

\section{Conclusion}

We observe $D^{0}-\bar{D}^{0}$ mixing with a significance equivalent to $6.1 \sigma$, by measuring the decaytime-dependence of the ratio of yields for the suppressed $D^{0} \rightarrow K^{+} \pi^{-}$to the favored $D^{0} \rightarrow K^{-} \pi^{+}$ decays using the full CDF data set. We measure the mixing parameters to be $R_{D}=(3.51 \pm 0.35) \times$ $10^{-3}, y^{\prime}=(4.3 \pm 4.3) \times 10^{-3}$, and $x^{\prime 2}=(0.08 \pm 0.18) \times 10^{-3}$. Our results are consistent with SM predictions and similar measurements from other experiments and substantially improve global knowledge of the charm mixing parameters.

\section{References}

[1] J. Beringer et al. (Particle Data Group), Phys. Rev. D 86, 010001 (2012)

[2] M. Starič et al. (Belle Collaboration), Phys. Rev. Lett. 98, 211803 (2007)

[3] B. Aubert et al. (BABAR Collaboration), Phys. Rev. Lett. 98, 211802 (2007)

[4] J.P. Lees et al. (BABAR Collaboration), Phys. Rev. D 87, 012004 (2013)

[5] T. Aaltonen et al. (CDF Collaboration), Phys. Rev. Lett. 100, 121802 (2008)

[6] LHCb Collaboration, R. Aaij et al. (LHCb Collaboration), Phys. Rev. Lett. 110, 101802 (2013)

[7] C. A. Chavez, R. F. Cowan, and W. S. Lockman, Int. J. Mod. Phys. A 27, 1230019 (2012)

[8] E. Golowich, J. Hewett, S. Pakvasa, and A. A. Petrov, Phys. Rev. D 76, 095009 (2007)

[9] D. Acosta et al. (CDF Collaboration), Phys. Rev. D 71, 032001 (2005)

[10] A. Abulencia et al. (CDF Collaboration), Phys. Rev. D 74, 031109(R) (2006) 